\title{
Probabilistic Justification Logic
}

\author{
Ioannis Kokkinis ${ }^{1}$, Zoran Ognjanović ${ }^{2}$, and Thomas Studer*3 \\ ${ }^{1}$ Department of Mathematics, Aristotle University of Thessaloniki, \\ Thessaloniki, Greece, ikokkinis@math.auth.gr \\ ${ }^{2}$ Mathematical Institute SANU, Belgrade, Serbia, \\ zorano@mi.sanu.ac.rs \\ ${ }^{3}$ Institute of Computer Science, University of Bern, Bern, \\ Switzerland, thomas.studer@inf.unibe.ch
}

\begin{abstract}
We present a probabilistic justification logic, PPJ, as a framework for uncertain reasoning about rational belief, degrees of belief and justifications. We establish soundness and strong completeness for PPJ with respect to the class of so-called measurable Kripke-like models and show that the satisfiability problem is decidable. We discuss how PPJ provides insight into the well-known lottery paradox.
\end{abstract}

Keywords: justification logic, probabilistic logic, strong completeness, decidability, lottery paradox

\section{Introduction}

In epistemic modal logic, we use formulas of the form $\square A$ to express that $A$ is believed. Justification logic unfolds the $\square$-modality into a family of so-called justification terms to represent evidence for an agent's belief. That is in justification logic we use $t: A$ to state that $A$ is believed for reason $t$.

Originally, Artemov developed the first justification logic, the Logic of Proofs, to give a classical provability semantics for intuitionistic logic $[1,2,6,20,21]$. Later, Fitting [9] introduced epistemic models for justification logic. As it turned out this interpretation provides a very successful approach to study many epistemic puzzles and problems $[3,7,19]$.

${ }^{*}$ Corresponding author 
In this paper, we extend justification logic with probability operators in order to accommodate the idea that

different kinds of evidence for $A$ lead to different degrees of belief in $A$.

In [15] we have introduced a first probabilistic justification logic PJ, which features formulas of the form $P_{\geq s}(t: A)$ to state that the probability of $t: A$ is greater than or equal to $s$. The language of $\mathrm{PJ}$, however, does neither include justification statements over probabilities (i.e. $\left.t:\left(P_{\geq s} A\right)\right)$ nor iterated probabilities (i.e. $\left.P_{\geq r}\left(P_{\geq s} A\right)\right)$.

In the present paper, we remedy these shortcomings and present the logic PPJ, which supports formulas of the form $t:\left(P_{\geq s} A\right)$ as well as $P_{\geq r}\left(P_{\geq s} A\right)$. This explains the name PPJ: the two $P$ 's refer to iterated $P$-operators. The necessity of allowing iterations of the probability operators can be seen when one wants to express a statement like "I am uncertain for a justification about the fact that a coin is counterfeit". While the previous statement cannot be expressed in the non-iterated probabilistic logic PJ it can be expressed nicely in PPJ by a formula of the form $P_{\geq s}\left(t: P_{\geq r} A\right)$ where $A$ is the event that a coin lands tails, $P_{\geq r} A$ is a formula expressing that the coin is counterfeit (for some $r>\frac{1}{2}$ ), $t$ is a justification for the fact that the coin is counterfeit and $r$ and $s$ are probabilities expressing our uncertainty for the event that the coin lands tails and for the fact that $t$ is a justification for the coin being counterfeit.

Our paper is organized as follows: In Section 3 we introduce syntax and semantics for PPJ and establish soundness and strong completeness. In Section 4 we show that satisfiability for PPJ is decidable and finally in Section 5 we present an application of PPJ to the lottery paradox.

Related work. The design of PPJ follows that of $\mathrm{LPP}_{1}$, which is a probability logic over classical propositional logic [27, 28]. The proofs that we present for PPJ are extensions of the corresponding proofs for LPP ${ }_{1}$. Note, however, that these extensions are non-trivial due to the presence of formulas of the form $t:\left(P_{\geq s} A\right)$.

As already mentioned, PJ [15] is the precursor of PPJ without iterations of probability operators. Kokkinis [14] shows that the satisfiability problem in PJ has the same complexity as the corresponding problem in the underlying justification logic and that the satisfiability problem in PPJ is PSPACE-complete, which is the usual suspect in modal logics.

Our probability logics are not compact. Consider the set

$$
T:=\left\{\neg P_{=0} A\right\} \cup\left\{P_{<1 / n} A \mid n \text { is a positive integer }\right\} .
$$

Although every finite subset of $T$ is satisfiable, the set $T$ is not. Hence in order to obtain a strong completeness result, we use an infinitary rule, which originates from $[27,29,30]$.

Milnikel [25] proposes a logic with uncertain justifications. We thoroughly study the relationship between Milnikel's logic and our approach in [15] where we show 
that three of his four axioms are theorems in our logic and that the fourth axiom holds under an additional independence assumption.

In $[11,12]$ Ghari presents fuzzy variants of justification logic, in which an agent can have a justification for a statement with certainty between 0 and 1 . He introduces fuzzy Fitting models and establishes a graded completeness theorem. Ghari also shows that Milnikel's principles are valid in his fuzzy setting.

Fan and Liau [8] introduce a possibilistic justification logic, which is an explicit version of a graded modal logic. Their logic includes formulas $t:_{r} A$ to express that according to evidence $t, A$ is believed with certainty at least $r$. However, the following principle holds in their logic:

$$
s:_{r} A \wedge t:_{q} A \rightarrow s:_{\max (r, q)} A .
$$

Hence all justifications for a belief yield the same (strongest) certainty, which is not in accordance with our guiding idea (1).

Artemov [5] studies a justification logic to formalize aggregated probabilistic evidence. His approach can handle conflicting and inconsistent data as well as positive and negative evidence for the same proposition.

\section{Funding}

Ioannis Kokkinis and Thomas Studer are supported by the SNSF project 153169, Structural Proof Theory and the Logic of Proofs. Zoran Ognjanovic is supported by the Serbian Ministry of Education, Science and Technological Development.

\section{The Probabilistic Justification Logic PPJ}

In this section we present syntax and semantics for the probabilistic justification logic PPJ.

\section{Syntax}

Justification terms are built from countably many constants and countably many variables according to the following grammar:

$$
t::=c|x|(t \cdot t)|(t+t)| ! t
$$

where $c$ is a constant and $x$ is a variable. Tm denotes the set of all terms and Con denotes the sets of all constants. For any term $t$ and natural number $n$ we define $!^{0} t:=t$ and $!^{n+1} t:=!\left(!^{n} t\right)$.

Let Prop be a countable set of atomic propositions. We will represent the set of all rational numbers with the symbol $\mathbb{Q}$. We define $\mathbb{Q}[0,1]:=\mathbb{Q} \cap[0,1]$, while $\mathbb{Q}[0, t)$ will denote the set $[0, t) \cap \mathbb{Q}[0,1]$. 
The set of formulas $\mathcal{L}$ is defined by the following grammar ${ }^{1}$ :

$$
A::=p\left|P_{\geq s} A\right| \neg A|A \wedge A| t: A
$$

where $t \in \operatorname{Tm}, s \in \mathbb{Q}[0,1]$ and $p \in$ Prop. We define the following abbreviations:

$$
\begin{aligned}
A \vee B & \equiv \neg(\neg A \wedge \neg B) \\
A \rightarrow B & \equiv \neg A \vee B \\
A \leftrightarrow B & \equiv(A \rightarrow B) \wedge(B \rightarrow A) \\
\perp & \equiv A \wedge \neg A, \text { for some } A \in \mathcal{L} \\
\top & \equiv A \vee \neg A, \text { for some } A \in \mathcal{L}
\end{aligned}
$$

Additionally, we set

$$
\begin{array}{rlrl}
P_{<s} A & \equiv \neg P_{\geq s} A & & P_{\leq s} A \equiv P_{\geq 1-s} \neg A \\
P_{>s} A \equiv \neg P_{\leq s} A & & P_{=s} A \equiv P_{\geq_{s}} A \wedge P_{\leq s} A
\end{array}
$$

The axiom schemes of PPJ are presented in Figure 1. Axiom (NN) corresponds to the fact that the probability of truthfulness of every formula is at least 0 (the acronym (NN) stands for non-negative). Observe that by substituting $\neg A$ for $A$ in (NN), we have $P_{\geq 0} \neg A$, which by our syntactical abbreviations is $P_{\leq 1} A$. Hence axiom (NN) also corresponds to the fact that the probability of truthfulness for every formula is at most 1. Axioms (L1) and (L2) describe some properties of inequalities (the L in (L1) and (L2) stands for less). Axioms (Add1) and (Add2) correspond to the additivity of probabilities for disjoint events (the Add in (Add1) and (Add2) stands for additivity).

\begin{tabular}{|cl|}
\hline (P) & finitely many schemes in the language of $\mathcal{L}$ \\
& axiomatizing classical propositional logic \\
$(\mathrm{J})$ & $\vdash u:(A \rightarrow B) \rightarrow(v: A \rightarrow u \cdot v: B)$ \\
$(+)$ & $\vdash u: A \vee v: A \rightarrow u+v: A$ \\
(NN) & $\vdash P_{\geq 0} A$ \\
$($ L1 $)$ & $\vdash P_{\leq r} A \rightarrow P_{<s} A$, where $s>r$ \\
(L2) & $\vdash P_{<s} A \rightarrow P_{\leq s} A$ \\
(Add1) & $\vdash P_{\geq r} A \wedge P_{\geq s} B \wedge P_{\geq 1} \neg(A \wedge B) \rightarrow P_{\geq \min (1, r+s)}(A \vee B)$ \\
(Add2) & $\vdash P_{\leq r} A \wedge P_{<s} B \rightarrow P_{<r+s}(A \vee B)$, where $r+s \leq 1$ \\
\hline
\end{tabular}

Figure 1: Axioms Schemes of PPJ

A constant specification is any set CS that satisfies

$$
\mathrm{CS} \subseteq\{(c, A) \mid c \text { is a constant and }
$$

$A$ is an instance of some axiom of PPJ $\}$.

\footnotetext{
${ }^{1}$ In order to have a countable language and obtain decidability, we restrict our probabilistic operators to the rational numbers.
} 
A constant specification CS is called:

axiomatically appropriate: if for every axiom instance $A$ of PPJ, there exists a constant $c$ such that $(c, A) \in \mathrm{CS}$;

schematic: if for every constant $c$, the set $\{A \mid(c, A) \in \mathrm{CS}\}$ consists of all instances of several (possibly zero) axiom schemes;

finite: if CS is a finite set;

decidable: if CS is a decidable set;

almost schematic: if $\mathrm{CS}=\mathrm{CS}_{1} \cup \mathrm{CS}_{2}$ where $\mathrm{CS}_{1} \cap \mathrm{CS}_{2}=\emptyset, \mathrm{CS}_{1}$ is schematic and $\mathrm{CS}_{2}$ is finite.

The notion of a schematic constant specification is crucial for establishing decidability of PPJ.

Let CS be any constant specification. The deductive system PPJ $\mathrm{JS}_{\mathrm{S}}$ is the Hilbert system obtained by adding to the axioms of PPJ the rules (MP), (PN), (ST) and (AN!) as given in Figure 2. Rule (PN) is the probabilistic analogue of the necessitation rule in modal logics (hence the acronym (PN) stands for probabilist necessitation): if a formula is valid, then it has probability 1 . Rule (ST) intuitively states that if the probability of a formula is arbitrary close to $s$, then it is at least $s$. The acronym (ST) stands for strenghtening. Note that (ST) is an infinitary rule, which we need to obtain strong completeness.

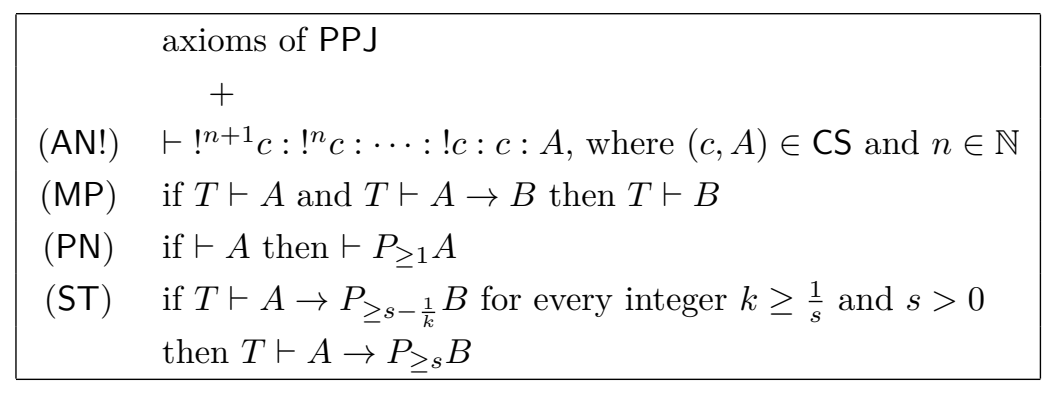

Figure 2: System PPJCS

A formula $A$ is deducible from a set $T$ of formulas $(T \vdash A)$ if there is an at most countable sequence of formulas $A_{0}, A_{1}, \ldots, A$ such that every $A_{i}$ is an axiom or a formula from the set $T$, or it is derived from the preceding formulas by an inference rule, with the exception that Rule (PN) can be applied on the theorems only. A formula $A$ is a theorem $(\vdash A)$ if it is deducible from the empty set. 


\section{Semantics}

To introduce semantics for PPJ $\mathrm{J}_{\mathrm{CS}}$, we begin with the notion of a basic evaluation, which is the cornerstone for many interpretations of justification logic $[4,18]$. In the following we use $\mathcal{P}(X)$ to denote the power set of a set $X$. We will also use the symbols $T$ and $F$ to represent the truth values true and false respectively.

Definition 1 (Basic Evaluation). Let CS be a constant specification. A basic evaluation for CS, or a basic CS-evaluation, is a function $*$ that maps atomic propositions to truth values and maps justification terms to subsets of $\mathcal{L}$, i.e.

$$
*: \text { Prop } \rightarrow\{\mathrm{T}, \mathrm{F}\} \quad \text { and } \quad *: \mathrm{Tm} \rightarrow \mathcal{P}(\mathcal{L}),
$$

such that for $u, v \in \mathrm{Tm}$, for $c \in$ Con and $A, B \in \mathcal{L}$ we have:

1. $\left(A \rightarrow B \in u^{*}\right.$ and $\left.A \in v^{*}\right) \Longrightarrow B \in(u \cdot v)^{*}$

2. $u^{*} \cup v^{*} \subseteq(u+v)^{*}$

3. if $(c, A) \in \mathrm{CS}$ then for all $n \in \mathbb{N}$ we have ${ }^{2}$ :

$$
!^{n-1} c: !^{n-2} c: \cdots: ! c: c: A \in\left(!^{n} c\right)^{*} .
$$

We usually write $t^{*}$ and $p^{*}$ instead of $*(t)$ and $*(p)$, respectively.

Definition 2 (Algebra over a Set). Let $W$ be a non-empty set and let $H$ be a non-empty subset of $\mathcal{P}(W)$. We call $H$ an algebra over $W$ iff the following hold:

- $W \in H$;

- $U, V \in H \Longrightarrow U \cup V \in H$;

- $U \in H \Longrightarrow W \backslash U \in H$.

Definition 3 (Finitely Additive Measure). Let $H$ be an algebra over $W$ and $\mu: H \rightarrow[0,1]$. We call $\mu$ a finitely additive measure iff the following hold:

1. $\mu(W)=1$;

2. for all $U, V \in H$ :

$$
U \cap V=\emptyset \quad \Longrightarrow \quad \mu(U \cup V)=\mu(U)+\mu(V) .
$$

Definition 4 (Probability Space). A probability space is a triple

$$
\text { Prob }=\langle W, H, \mu\rangle,
$$

where:

\footnotetext{
${ }^{2}$ We agree to the convention that the formula $!^{n-1} c: !^{n-2} c: \cdots: ! c: c: A$ represents the formula $A$ for $n=0$.
} 
- $W$ is a non-empty set;

- $H$ is an algebra over $W$;

- $\mu: H \rightarrow[0,1]$ is a finitely additive measure.

Definition 5 (Model). Let CS be a constant specification. A PPJ $\mathrm{CS}_{\text {-model }}$ is a quintuple $M=\langle U, W, H, \mu, *\rangle$ where:

1. $U$ is a non-empty set of objects called worlds;

2. $W, H, \mu$ and $*$ are functions, which have $U$ as their domain, such that for every $w \in U$ :

- $\langle W(w), H(w), \mu(w)\rangle$ is a probability space with $W(w) \subseteq U$;

- $*_{w}$ is a basic CS-evaluation ${ }^{3}$.

The ternary satisfaction relation $\models$ is defined between models, worlds, and formulas.

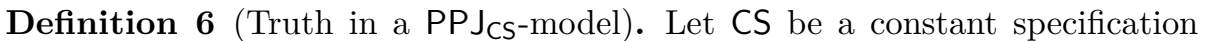
and let $M=\langle U, W, H, \mu, *\rangle$ be a PPJ $\mathrm{CS}_{\mathrm{CS}}$-model. We define what it means for an $\mathcal{L}$-formula to hold in $M$ at a world $w \in U$ inductively as follows:

$$
\begin{array}{rlll}
M, w \models p & : \Longleftrightarrow & p_{w}^{*}=\mathrm{T} \quad \text { for } p \in \text { Prop } \\
M, w \models P_{\geq s} B & : \Longleftrightarrow \quad\left([B]_{M, w} \in H(w) \text { and } \mu(w)\left([B]_{M, w}\right) \geq s\right) \\
& & \text { where }[B]_{M, w}=\{x \in W(w) \mid M, x \models B\} \\
M, w \models \neg B & : \Longleftrightarrow \quad M, w \not \models B \\
M, w \models B \wedge C & : \Longleftrightarrow & (M, w \models B \text { and } M, w \models C) \\
M, w \models t: B & : \Longleftrightarrow & B \in t_{w}^{*}
\end{array}
$$

Definition 7 (Measurable Model). Let CS be a constant specification and let $M=\langle U, W, H, \mu, *\rangle$ be a $\mathrm{PPJ}_{\mathrm{CS}}$-model. $M$ is called measurable iff for every $w \in U$ and for every $A \in \mathcal{L}$ :

$$
[A]_{M, w} \in H(w) .
$$

$P P J_{C S, \text { Meas }}$ denotes the class of $P P J_{C S}$-measurable models.

Let $T \subseteq \mathcal{L}$. Then $M, w \models T$ means that $M, w \models A$ for all $A \in T$. Further $T \models A$ means that for all $M \in \mathrm{PPJ}_{\mathrm{CS}, \text { Meas }}$ and for every world $w$ of $M, M, w \models T$ implies $M, w \models A$.

To be precise we should write $T \vdash_{\mathrm{CS}} A$ and $T \models_{\mathrm{CS}} A$ instead of $T \vdash A$ and $T \models A$, respectively, since these two notions depend on a given constant specification CS. However, CS will always be clear from the context and thus can be omitted.

\footnotetext{
${ }^{3}$ We will usually write $*_{w}$ instead of $*(w)$.
} 
Definition 8 (Satisfiability). We say a formula $A$ of $\mathcal{L}$ is satisfiable if there exist a PPJ $\mathrm{CS}_{\mathrm{C} \text { Meas }}$-model $M=\langle U, W, H, \mu, *\rangle$ and $w \in U$ with $M, w \models A$. A set of formulas $T$ is satisfiable if there is a world $w$ from a model $M$ such that every formula from $T$ holds in $M$ at $w$.

The following lemma was proved for the probabilistic justification logic PJ in [15]. The same proof also works in PPJ.

Lemma 9. For any $\mathrm{PPJ}_{\mathrm{CS}}$ we have:

$$
\vdash A \rightarrow B \quad \Longrightarrow \quad \vdash P_{\geq_{s}} A \rightarrow P_{\geq_{s}} B .
$$

We established the Deduction Theorem for PJ in [15]. Now we present the version for PPJ, which can be proved in the same way.

Theorem 10 (Deduction Theorem). Let $T \subseteq \mathcal{L}$ and $A, B \in \mathcal{L}$. For any constant specification CS we have:

$$
T, A \vdash B \quad \Longleftrightarrow \quad T \vdash A \rightarrow B .
$$

\section{Soundness and Completeness}

As usual, we can establish soundness by induction on the depth of the derivation of a formula $A$.

Theorem 11 (Soundness). For any constant specification CS, PPJCS is sound with respect to the class of $\mathrm{PPJ}_{\mathrm{CS}, \text { Meas }}$-models. I.e. for any $A \in \mathcal{L}$ and $T \subseteq \mathcal{L}$ we have:

$$
T \vdash A \quad \Longrightarrow \quad T \models A \text {. }
$$

The completeness proof for $P P J_{C S}$ is a combination of the completeness proof for $\mathrm{LPP}_{1}$ [28] and the completeness proof for PJ [15]. In the rest of the section we are going to present a series of definitions and lemmata that lead to the strong completeness theorem for PPJ. When the proofs are very similar to the ones for PJ we will simply provide a reference to [15].

First we need the notion of a $\mathrm{PPJ}_{\mathrm{CS}}$-consistent set.

Definition 12 (PPJ $\mathrm{CS}_{\mathrm{C}-\text { consistent }}$ Set). Let $\mathrm{CS}$ be a constant specification and let $T$ be a set of $\mathcal{L}$-formulas.

- $T$ is said to be $P P J_{C S}$-consistent iff $T \nvdash \perp$. Otherwise $T$ is said to be $\mathrm{PPJ}_{\mathrm{CS}}$-inconsistent.

- $T$ is said to be maximal iff for every $A \in \mathcal{L}$ either $A \in T$ or $\neg A \in T$.

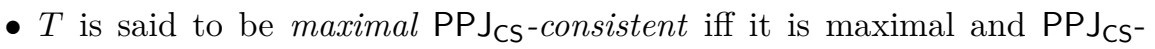
consistent. 
The next two lemmata state some standard properties of PPJ $\mathrm{CS}_{\mathrm{CS}}$-consistent sets. Both lemmata have been proved in [15] for the logic PJ. The proofs for PPJ are similar. Lemma 13 is essential for the construction of maximal consistent sets. Its second claim is needed to deal with the rule (ST).

Lemma 13 (Properties of PPJ $\mathrm{CS}$-Consistent Sets). Let CS be a constant speci-

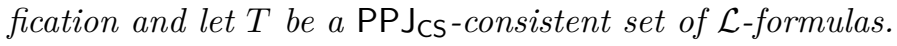

(1) For any formula $A \in \mathcal{L}$ we have that $T, A$ is $\mathrm{PPJ}_{\mathrm{CS}}$-consistent or $T, \neg A$ is $\mathrm{PPJ}_{\mathrm{CS}-\text { consistent. }}$

(2) If $\neg\left(A \rightarrow P_{\geq s} B\right) \in T$ for $s>0$, then there is some integer $n \geq \frac{1}{s}$ such that $T, \neg\left(A \rightarrow P_{\geq s-\frac{1}{n}} B\right)$ is $\mathrm{PPJ}_{\mathrm{CS}}$-consistent.

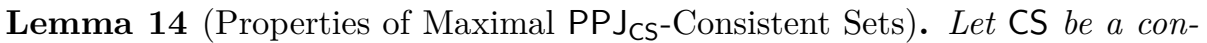
stant specification and let $\mathcal{T}$ be a maximal $\mathrm{PPJ}_{\mathrm{CS}}$-consistent set. Then the following hold:

(1) For any formula $A \in \mathcal{L}$, exactly one member of $\{A, \neg A\}$ is in $\mathcal{T}$.

(2) For any formula $A \in \mathcal{L}$ :

$$
\mathcal{T} \vdash A \Longleftrightarrow A \in \mathcal{T}
$$

(3) For all formulas $A, B \in \mathcal{L}$ we have:

$$
A \vee B \in \mathcal{T} \Longleftrightarrow A \in \mathcal{T} \text { or } B \in \mathcal{T}
$$

(4) For all formulas $A, B \in \mathcal{L}$ we have:

$$
A \wedge B \in \mathcal{T} \Longleftrightarrow\{A, B\} \subseteq \mathcal{T}
$$

(5) For all formulas $A, B \in \mathcal{L}$ we have:

$$
\{A, A \rightarrow B\} \subseteq \mathcal{T} \Longrightarrow B \in \mathcal{T}
$$

(6) Let $A \in \mathcal{L}, X=\left\{s \mid P_{\geq s} A \in \mathcal{T}\right\}$ and $t=\sup (X)$. Then:

(i) For all $r \in \mathbb{Q}[0, t)$ we have that $P_{>r} A \in \mathcal{T}$

(ii) For all $r \in \mathbb{Q}[0, t)$ we have that $P_{\geq_{r}} A \in \mathcal{T}$

(iii) If $t \in \mathbb{Q}[0,1]$ then $P_{\geq t} A \in \mathcal{T}$.

(iv) For any $r \in \mathbb{Q}[0,1]$ :

$$
t \geq r \Longleftrightarrow P_{\geq_{r}} A \in \mathcal{T}
$$

As the next step we have to show that Lindenbaum lemma holds for the logic PPJ. 
Lemma 15 (Lindenbaum). Let CS be a constant specification. Every PPJ $\mathrm{CS}^{-}$ consistent set can be extended to a maximal $\mathrm{PPJ} \mathrm{CS}_{\mathrm{C}-\text { consistent set. }}$

Proof. This lemma has been proved for the logic PJ in [15] and the proof for PPJ is similar. However, it is worth highlighting that the proof of the Lindenbaum lemma for the logics PJ and PPJ is much more complex than usual. The reason is that the logics PJ and PPJ can have proofs of infinite depth because of the rule (ST). We briefly explain the amendments that need to be done to the usual proof for Lindenbaum lemma.

The typical construction of the maximal consistent set in Lindenbaum lemma is as follows:

Let $T$ be a PPJCS-consistent set. Let $A_{0}, A_{1}, A_{2}, \ldots$ be an enumeration of all formulas in $\mathcal{L}$. We define a sequence of sets $\left\{T_{i}\right\}_{i \in \mathbb{N}}$ such that:

(1) $T_{0}:=T$

(2) for every $i \geq 0$ :

(a) if $T_{i} \cup\left\{A_{i}\right\}$ is $\mathrm{PJ}_{\mathrm{CS}}$-consistent, then we set $T_{i+1}:=T_{i} \cup\left\{A_{i}\right\}$, otherwise

(b) we set $T_{i+1}:=T_{i} \cup\left\{\neg A_{i}\right\}$

(3) $\mathcal{T}=\bigcup_{i=0}^{\infty} T_{i}$

It is then straightforward to show that $\mathcal{T}$ is $\mathrm{PPJ}_{\mathrm{CS}}$-maximal and that every $T_{i}$ is $\mathrm{PPJ}_{\mathrm{CS}}$-consistent. However the consistency of every $T_{i}$ does not imply the consistency of $\mathcal{T}$, since proofs in PJ and PPJ may have infinite depth. We can tackle this problem by adding the formula $\neg\left(B \rightarrow P_{\geq s-\frac{1}{n}} C\right)$ to $T_{i+1}$ for a suitable $n$, when $A_{i}$ is of the form $B \rightarrow P_{\geq s} C$ and $T_{i} \cup\left\{A_{i}\right\}$ is not $\mathrm{PJ}_{\mathrm{CS}^{-}}$ consistent. Lemma 13 guarantees the existence of such an $n$.

Now we define a canonical model for any maximal PPJ $\mathrm{CS}_{\mathrm{CS}}$-consistent set of formulas.

Definition 16 (Canonical Model). Let CS be a constant specification. The canonical model for PPJ $\mathrm{PSS}_{\mathrm{C}}$ is given by the quintuple $M=\langle U, W, H, \mu, *\rangle$, defined as follows:

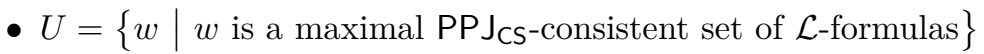

- for every $w \in U$ the probability space $\langle W(w), H(w), \mu(w)\rangle$ is defined as follows:

1. $W(w)=U$

2. $H(w)=\left\{(A)_{M} \mid A \in \mathcal{L}\right\}$

where $(A)_{M}=\{x \mid x \in U, A \in x\}$. If $M$ is clear from the context, we may simply write $(A)$ instead of $(A)_{M}$.

3. for all $A \in \mathcal{L}, \mu(w)\left((A)_{M}\right)=\sup _{s}\left\{P_{\geq s} A \in w\right\}$ 
- for every $w \in W$ the basic CS-evaluation $*_{w}$ is defined as follows:

1. for all $p \in$ Prop:

$$
p_{w}^{*}= \begin{cases}\mathrm{T} & \text { if } p \in w \\ \mathrm{~F} & \text { if } \neg p \in w\end{cases}
$$

2. for all $t \in \mathrm{Tm}$ :

$$
t_{w}^{*}=\{A \mid t: A \in w\}
$$

The following properties of the set $(A)_{M}$ are direct consequences of Lemma 14 .

Lemma 17. Let $M=\langle U, W, H, \mu, *\rangle$ be the canonical model for some PPJ $J_{\mathrm{CS}}$ and let $A, B \in \mathcal{L}$. Then the following hold:

(i) $(\neg A)_{M}=U \backslash(A)_{M}$

(ii) $(A)_{M} \cap(B)_{M}=(A \wedge B)_{M}$

(iii) $(A)_{M} \cup(B)_{M}=(A \vee B)_{M}$

Now we will prove that the canonical model for PPJ $\mathrm{JSS}_{\mathrm{CS}}$ is a $\mathrm{PPJ} \mathrm{JS}_{\mathrm{CS}}$-model.

Lemma 18. Let CS be a constant specification. The canonical model for PPJ $\mathrm{JS}_{\mathrm{CS}}$ is a $\mathrm{PPJ} \mathrm{CS}_{\text {-model. }}$

Proof. Let $M=\langle U, W, H, \mu, *\rangle$ be the canonical model for PPJ $J_{\mathrm{CS}}$. In order for $M$ to be a PPJ $\mathrm{CS}$-model we have to prove the following:

$U$ is a non-empty set:

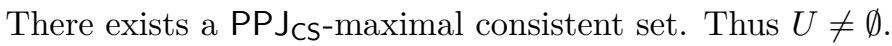

For every $w \in U$ the triple $\langle W(w), H(w), \mu(w)\rangle$ is a probability space:

We have to prove the following:

(1) $\boldsymbol{W}(\boldsymbol{w})$ is a non-empty subset of $\boldsymbol{U}$

It is obvious since $W(w)=U$ and $U \neq \emptyset$.

(2) $\boldsymbol{H}(\boldsymbol{w})$ is an algebra over $\boldsymbol{W}(\boldsymbol{w})$

It holds that:

$$
(\top)_{M}=\{x \mid x \in U, \top \in x\}=U=W(w) .
$$

Thus $W(w) \in H(w)$.

Let $(A)_{M} \in H(w)$ for some $A \in \mathcal{L}$. It holds that:

$$
(A)_{M}=\{x \mid x \in U, A \in x\} \subseteq U=W(w) .
$$

Thus $H(w) \subseteq \mathcal{P}(W(w))$.

Let $(A),(B) \in H(w)$ for some $A, B \in \mathcal{L}$. By Lemma 17 we have that $W(w) \backslash(A)=U \backslash(A)=(\neg A) \in H(w)$ and $(A) \cup(B)=(A \vee B) \in H(w)$. So, according to Definition 2, $H(w)$ is an algebra over $W(w)$. 
(3) $\mu(w)$ is a function from $H(w)$ to $[0,1]$ :

We have to prove the following:

(a) the domain of $\mu(w)$ is $H(w)$ and the codomain of $\mu(w)$ is $[0,1]$ :

Let $(A) \in H(w)$. We have that $P_{\geq 0} A$ is an axiom of PPJ, thus $P_{\geq 0} A \in w$. Hence the set $\left\{s \in S \mid P_{\geq s} A \in w\right\}$ is not empty which means that it has a supremum. So $\mu(w)((A))$ is defined. Thus the domain of $\mu(w)$ is $H(w)$.

Let $(A) \in H(w)$. We have that $\mu(w)((A))=\sup _{s}\left\{P_{\geq_{s}} A \in w\right\} \geq 0$. In $\sup _{s}\left\{P_{\geq_{s}} A \in w\right\}$ we have by definition that $s \in \mathbb{Q}[0,1]$, i.e. $s \leq 1$. Thus $\sup _{s}\left\{P_{\geq s} A \in w\right\} \leq 1$, i.e. $\mu(w)((A)) \leq 1$. So the codomain of $\mu(w)$ is $[0,1]$.

(b) for every $\boldsymbol{V} \in \boldsymbol{H}(\boldsymbol{w}), \boldsymbol{\mu}(\boldsymbol{w})(\boldsymbol{V})$ is unique:

Let $V \in H(w)$ and assume that $V=(A)=(B)$ for some $A, B \in \mathcal{L}$. We will prove that $\mu(w)((A))=\mu(w)((B))$. Of course it suffices to prove that:

$$
(A) \subseteq(B) \Longrightarrow \mu(w)((A)) \leq \mu(w)((B))
$$

We have:

$$
\begin{array}{cr}
(A) \subseteq(B) & \text { implies } \\
(\forall x \in U)[x \in(A) \Longrightarrow x \in(B)] & \text { implies } \\
(\forall x \in U)[A \in x \Longrightarrow B \in x] & \text { implies } \\
(\forall x \in U)[A \notin x \text { or } B \in x] & \text { implies by L. 14(1) } \\
(\forall x \in U)[\neg A \in x \text { or } B \in x] & \text { implies by L. 14(3) } \\
(\forall x \in U)[\neg A \vee B \in x] & \text { implies } \\
(\forall x)\left[x \text { is a maximal PP } \text { CS-consistent set }_{\text {r }}\right. & \\
\Longrightarrow A \rightarrow B \in x] &
\end{array}
$$

Assume that $\nvdash A \rightarrow B$. By propositional reasoning we get $\nvdash \neg(A \rightarrow$

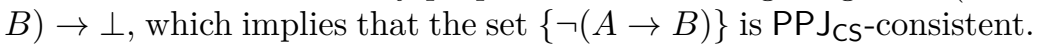
By Lemma 15 we have that there exists a maximal PPJ $\mathrm{CS}^{-}$-consistent set $\mathcal{T}$ such that $\mathcal{T} \supseteq\{\neg(A \rightarrow B)\}$. However by (3) we have that $A \rightarrow B \in \mathcal{T}$ which contradicts the fact that $\mathcal{T}$ is $\mathrm{PPJ}_{\mathrm{CS}}$-consistent. Thus $\vdash A \rightarrow B$. As a consequence, by Lemma 9, we have that

$$
(\forall s \in \mathbb{Q}[0,1])\left[\vdash P_{\geq s} A \rightarrow P_{\geq s} B\right] .
$$

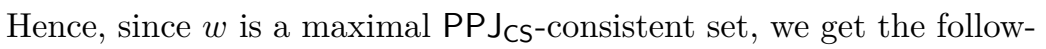


ing:

$$
\begin{array}{lr}
(\forall s \in \mathbb{Q}[0,1])\left[P_{\geq s} A \rightarrow P_{\geq s} B \in w\right] & \text { implies by L. 14(5) } \\
(\forall s \in \mathbb{Q}[0,1])\left[P_{\geq s} A \in w \Longrightarrow P_{\geq s} B \in w\right] & \text { implies } \\
\left\{s \in \mathbb{Q}[0,1] \mid P_{\geq s} A \in w\right\} \subseteq\left\{s \in \mathbb{Q}[0,1] \mid P_{\geq s} B \in w\right\} & \text { implies } \\
\sup _{s}\left\{P_{\geq s} A \in w\right\} \leq \sup _{s}\left\{P_{\geq s} B \in w\right\} & \text { i.e. } \\
\mu(w)((A)) \leq \mu(w)((B)) &
\end{array}
$$

Hence (2) holds, which proves that $\mu(w)(V)$ is unique.

\section{(4) $\boldsymbol{\mu}(\boldsymbol{w})$ is a finitely additive measure:}

This case has been proved in [15] for the logic PJ. This proof also works for PPJ.

For every $\boldsymbol{w} \in \boldsymbol{W} *_{\boldsymbol{w}}$ is a basic CS-evaluation:

This holds by the construction of the canonical model

The fact that the canonical model is measurable will be a corollary of the next lemma.

Lemma 19. Let $M=\langle U, W, H, \mu, *\rangle$ be the canonical model for PPJ $\mathrm{J}_{\mathrm{CS}}$. Then we have

$$
(\forall A \in \mathcal{L})(\forall w \in U)\left[[A]_{M, w}=(A)_{M}\right]
$$

Proof. Let $w \in U$ and let $A \in \mathcal{L}$. We will prove the claim by induction on the complexity of $A$. We distinguish the following cases:

1. $A \equiv p \in$ Prop. It holds that:

$$
\begin{aligned}
{[A]_{M, w}=[p]_{M, w} } & =\{x \in W(w) \mid M, x \models p\}=\left\{x \in U \mid p_{x}^{*}=\mathrm{T}\right\} \\
& =\{x \in U \mid p \in x\}=(p)_{M}=(A)_{M} .
\end{aligned}
$$

2. $A \equiv t: B$. It holds that:

$$
\begin{aligned}
{[A]_{M, w}=[t: B]_{M, w} } & =\{x \in W(w) \mid M, x \models t: B\}=\left\{x \in U \mid B \in t_{x}^{*}\right\} \\
& =\{x \in U \mid t: B \in x\}=(t: B)_{M}=(A)_{M} .
\end{aligned}
$$

3. $A \equiv P_{\geq s} B$. By i.h. we have that for all $x \in U,[B]_{M, x}=(B)_{M} \in H(x)$. Thus, we have:

$$
\begin{aligned}
{[A]_{M, w}=\left[P_{\geq s} B\right]_{M, w} } & =\left\{x \in W(w) \mid M, x \models P_{\geq s} B\right\} \\
& =\left\{x \in W(w) \mid \mu(x)\left([B]_{M, x}\right) \geq s\right\} \\
& =\left\{x \in W(w) \mid \mu(x)\left((B)_{M}\right) \geq s\right\} \\
& =\left\{x \in U \mid \sup _{r}\left\{P_{\geq r} B \in x\right\} \geq s\right\}
\end{aligned}
$$


By Lemma 14(6)(iv) we get:

$$
[A]_{M, w}=\left\{x \in U \mid P_{\geq_{s}} B \in x\right\}=\left(P_{\geq_{s}} B\right)_{M}=(A)_{M} .
$$

4. $A \equiv B \wedge C$. It holds that:

$$
\begin{aligned}
{[A]_{M, w} } & =[B \wedge C]_{M, w}=[B]_{M, w} \cap[C]_{M, w} \stackrel{\text { i.h. }}{=}(B)_{M, w} \cap(C)_{M, w} \\
& \stackrel{L}{ } \stackrel{17(i i)}{=}(B \wedge C)_{M, w}=(A)_{M} .
\end{aligned}
$$

5. $A \equiv \neg B$. It holds that:

$$
\begin{aligned}
{[A]_{M, w} } & =[\neg B]_{M, w}=W(w) \backslash[B]_{M, w} \stackrel{\text { i.h. }}{=} U \backslash(B)_{M, w} \\
& \stackrel{L}{=}{ }^{=}(\neg B)_{M, w}=(A)_{M} .
\end{aligned}
$$

From Lemmata 18 and 19 we get the following corollary.

Corollary 20. Let CS be any constant specification. The canonical model for $\mathrm{PPJ}_{\mathrm{CS}}$ is a $\mathrm{PPJ} \mathrm{JS}_{\text {, Meas }}$-model.

Proof. Let $M=\langle U, W, H, \mu, *\rangle$ be the canonical model for PPJ $\mathrm{JS}_{\mathrm{CS}}$ and let $A \in \mathcal{L}$.

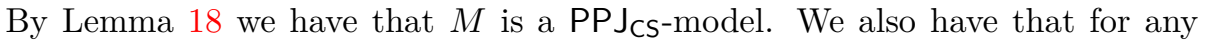
$w \in U$, by Lemma 19, $[A]_{M, w}=(A)_{M} \in H(w)$. Thus, $M \in \operatorname{PPJ}_{\mathrm{CS}, \text { Meas }}$.

Making use of the properties of maximal consistent sets, we can now establish the Truth Lemma.

Lemma 21 (Truth Lemma). Let CS be some constant specification and let $M=\langle U, W, H, \mu, *\rangle$ be the canonical model for PPJ $\mathrm{CS}_{\mathrm{CS}}$ For every $A \in \mathcal{L}$ and any $w \in U$ we have:

$$
A \in w \quad \Longleftrightarrow \quad M, w \models A .
$$

Proof. We prove the claim by induction on the structure of $A$. Let us only show the case for $A=P_{\geq s} B$. It holds:

$$
\begin{array}{ll}
M, w \models A & \Longleftrightarrow \\
M, w \models P_{\geq s} B & \Longleftrightarrow \\
\mu(w)\left([B]_{M, w}\right) \geq s & \stackrel{L^{L .19}}{\Longleftrightarrow} \\
\mu(w)\left((B)_{M}\right) \geq s & \stackrel{D .16}{\Longleftrightarrow} \\
\sup _{r}\left\{P_{\geq r} B \in w\right\} \geq s & \stackrel{L .}{\Longleftrightarrow} \\
P_{\geq s} B \in w & \Longleftrightarrow \\
A \in w &
\end{array}
$$


Finally, we get the strong completeness theorem as usual.

Theorem 22 (Strong Completeness for PPJ). Let CS be a constant specification, let $T \subseteq \mathcal{L}$ and let $A \in \mathcal{L}$. Then we have:

$$
T \models A \quad \Longrightarrow \quad T \vdash A .
$$

Proof. We prove the claim by contraposition. Assume that $T \nvdash A$. This means that $T \nvdash(\neg A) \rightarrow \perp$, By Theorem 10 we get $T, \neg A \nvdash \perp$, i.e. the set $T \cup\{\neg A\}$

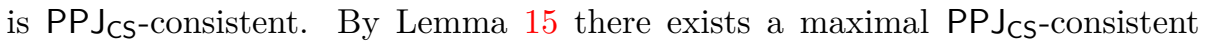
set $w$, such that $w \supseteq T \cup\{\neg A\}$. Let $M$ be the canonical model for PPJ $J_{C S}$. By Corollary 20 we have that $M \in \mathrm{PPJ}_{\mathrm{CS} \text {, Meas }}$. By Lemma 21 we have that $M, w \models T$ and $M, w \models \neg A$. Hence $T \not \models A$.

\section{Decidability for PPJ}

Assume that we want to test some $A \in \mathcal{L}$ for satisfiability. The test is divided in two parts: first we test whether the "justification and classical constraints" that appear in $A$ are satisfiable and then we test whether the "probabilistic constraints" that appear in $A$ are satisfiable. Of course we have to make sure that both kinds of constraints are satisfied in the same PPJ $\mathrm{CS}_{\mathrm{S} \text {,Meas }}$-model. The satisfiability testing for the "justification and classical constraints" will be done using an adaptation of the satisfiability algorithm for the logic J ([16, 17, 26, 31]), whereas the satisfiability testing for the "probabilistic constraints" will be done using similar ideas as the ones used for the satisfiability testing in the logic PJ [13]. In order to formally present our satisfiability algorithm we will first explain what is meant under "satisfiability testing for justification and classical constraints", then what is formally meant under "satisfiability testing for probabilistic constraints" and finally how both kind of constraints can be satisfied at the same model.

By testing satisfiability of "justification and classical constraints" that appear in an $\mathcal{L}$-formula we mean that we test whether a CS-evaluation satisfies an $\mathcal{L}$ formula. In order to formally define the sentence "a CS-evaluation satisfies an $\mathcal{L}$-formula" we have to extend the definition of a CS-evaluation.

Definition 23 (Extended Basic CS-Evaluation). Let CS be any constant specification. An extended basic CS-evaluation, is a function $*$ that maps atomic propositions and $\mathcal{L}$-formulas of the form $P_{\geq s} A$ to truth values and maps justification terms to sets of $\mathcal{L}$-formulas such that the conditions of Definition 1 are satisfied. That is for $p \in$ Prop, $u, v \in \operatorname{Tm}, c \in$ Con, $A, B \in \mathcal{L}$ and $s \in \mathbb{Q}[0,1]$ we have:

(1) $\left(P_{\geq s} A\right)^{*} \in\{\mathrm{T}, \mathrm{F}\}, p^{*} \in\{\mathrm{T}, \mathrm{F}\}$ and $u^{*} \subseteq \mathcal{L}$;

(2) $\left(A \rightarrow B \in u^{*}\right.$ and $\left.A \in v^{*}\right) \Longrightarrow B \in(u \cdot v)^{*}$; 
(3) $u^{*} \cup v^{*} \subseteq(u+v)^{*}$;

(4) if $(c, A) \in \mathrm{CS}$ then for all $n \in \mathbb{N}$ we have:

$$
!^{n-1} c: !^{n-2} c: \cdots: ! c: c: A \in\left(!^{n} c\right)^{*} .
$$

Satisfiability under an extended basic CS-evaluation can be defined in the following way:

Definition 24 (Satisfiability under an Extended Basic CS-evaluation). Let CS be a constant specification and let $*$ be some extended basic CS-evaluation. We define what it means for an $\mathcal{L}$-formula to hold in $*$ as follows:

$$
\begin{aligned}
* \models p & \Longleftrightarrow p^{*}=\mathrm{T} \quad \text { for } p \in \text { Prop } ; \\
* \models P & \Longleftrightarrow\left(P_{\geq s} B\right)^{*}=\mathrm{T} ; \\
* \models \neg B & \Longleftrightarrow \quad * B ; \\
* \models B \wedge C & \Longleftrightarrow \quad(* \models B \text { and } * \models C) ; \\
* \models t: B & \Longleftrightarrow B \in t^{*} .
\end{aligned}
$$

When we say that some $A \in \mathcal{L}$ is CS-satisfiable, we mean that there exists an extended basic CS-evaluation that satisfies $A$.

According to Definition 24 the satisfiability of an $\mathcal{L}$ formula under an extended basic CS-evaluation is similar to the satisfiability of a justification logic formula under a basic CS-evaluation [4]. Therefore, it makes sense to use an extension of the usual decision procedure for the basic justification logic $\mathrm{J}$ to decide whether an $\mathcal{L}$-formula is CS-satisfiable.

Before presenting the decidability algorithm for the logic PPJ, we briefly recall the decidability algorithm for the logic J. Let $A$ be a formula of justification logic and let CS be a constant specification. The key point in the satisfiability algorithm for the logic $\mathrm{J}$ is to decide whether some $t: B$ that appears in $A$ holds, i.e. whether $t$ justifies $B$. In general, this problem is undecidable since a given term may justify infinitely many formulas.

This problem can be solved by restricting the constant specification to be schematic. If we use schematic variables for formulas, then we can represent a schematic constant specification in a finite way, which also makes the number of (schematic) formulas that are justified by a term finite. The decision algorithm for the logic $\mathrm{J}$ also requires finding all formulas that are instances of two formula schemes, i.e. unifying two formula schemes. This question is naturally answered by finding the most general unifier of the two schemes.

Lemma 25. Let CS be a decidable and almost schematic constant specification. For any formula $A \in \mathcal{L}$, it is decidable whether $A$ is CS-satisfiable.

Proof. As mentioned earlier we can test whether an $\mathcal{L}$-formula is CS-satisfiable by extending the decidability algorithm for justification logic J. Most of the 
algorithm can be easily adapted to our probabilistic setting. The only part of the algorithm that needs major adaptations is the representation of schematic formulas and therefore the unification algorithm.

In the setting of PPJ we need three kinds of schematic variables: for terms, formulas and rational numbers. Because of the side conditions that come with the axioms (L1) and (Add2) our schematic formulas should be paired with systems of linear inequalities. For example, the scheme (L1) should be represented by the schematic formula $P_{\leq r} A \rightarrow P_{<s} A$ (with the schematic variables $r, s$, and $A$ ) together with the inequality $r<s$, whereas a scheme that is obtained by a conjunction of the schemes (L1) and (Add2) should be represented as

$$
\left(P_{\leq r_{1}} A_{1} \rightarrow P_{<s_{1}} A_{1}\right) \wedge\left(P_{\leq r_{2}} A_{2} \wedge P_{<s_{2}} B_{2} \rightarrow P_{<r_{2}+s_{2}}\left(A_{2} \vee B_{2}\right)\right)
$$

together with the inequalities

$$
\left\{r_{1}<s_{1}, r_{2}+s_{2} \leq 1\right\} .
$$

We should not forget that the rational variables belong to $\mathbb{Q}[0,1]$. So we have to add constraints like $0 \leq r \leq 1$.

Hence in addition to constructing a substitution, the unification algorithm also has to take care of the linear constraints. For instance, in order to unify the schemes $P_{\geq r} A$ and $P_{\geq s} B$ the algorithm has to unify $A$ and $B$, and to equate $r$ and $s$, i.e. it adds $r=s$ to the linear system. In the end, the constructed substitution only is a most general unifier if the linear system is satisfiable.

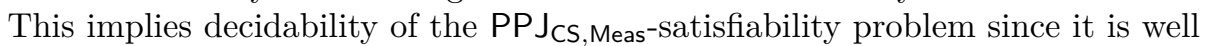
known that satisfiability testing for systems of linear equations is decidable (see e.g. [24]).

Another complication are constraints of the form

$$
l=\min (1, r+s)
$$

that originate from the scheme (Add1). Obviously, Eq. (4) is not linear. However, for a linear system $C$, we find that

$$
C \cup\{l=\min (1, r+s)\}
$$

has a solution if and only if

$$
C \cup\{l=r+s, r+s \leq 1\} \text { or } C \cup\{l=1, r+s>1\}
$$

has a solution. Thus we can reduce solving a system involving Eq. (4) to solving several linear systems.

Lemma 25 is enough for testing whether "justification and classical constraints" can be satisfied. Now we proceed with definitions and lemmata that are needed for testing the satisfiability of "probabilistic constraints". 
Definition 26 (Subformulas and Atoms). The set of subformulas, $\operatorname{subf}(\cdot)$, of an $\mathcal{L}$-formula is recursively defined by:

$$
\begin{aligned}
\operatorname{subf}(p) & :=\{p\} \quad \text { for } p \in \operatorname{Prop} ; \\
\operatorname{subf}\left(P_{\geq s} A\right) & :=\left\{P_{\geq s} A\right\} \cup \operatorname{subf}(A) ; \\
\operatorname{subf}(\neg A) & :=\{\neg A\} \cup \operatorname{subf}(A) ; \\
\operatorname{subf}(A \wedge B) & :=\{A \wedge B\} \cup \operatorname{subf}(A) \cup \operatorname{subf}(B) ; \\
\operatorname{subf}(t: A) & :=\{t: A\} \cup \operatorname{subf}(A) .
\end{aligned}
$$

Assume that $\operatorname{subf}(A)=\left\{A_{1}, \ldots, A_{k}\right\}$ for some $A \in \mathcal{L}$. A formula of the form

$$
\pm A_{1} \wedge \ldots \wedge \pm A_{k}
$$

where $\pm A_{i}$ is either $A_{i}$ or $\neg A_{i}$, will be called an atom of $A$. The set atoms $(A)$ contains all atoms of $A$.

Let $C \in \operatorname{atoms}(A)$ for some $A$. Then $B \in C$ means that $B$ is a subformula of $A$ that appears in $C$ (either negated or not).

Lemma 27. Let $M=\langle U, W, H, \mu, *\rangle \in \mathrm{PPJ}_{\mathrm{CS}, \text { Meas }}$ and let $A \in \mathcal{L}$. Further, let $B \in \operatorname{subf}(A)$, let $C \in \operatorname{atoms}(A)$ and let $w \in U$. Assume that $M, w \models C$. Then we have:

$$
M, w \models B \quad \Longleftrightarrow \quad B \in C .
$$

Proof. We prove the two directions of the lemma separately:

$\Longleftarrow$ : From $B \in C$ and $M, w \models C$ we immediately get $M, w \models B$.

$\Longrightarrow$ : Since $B$ is a subformula of $A$, we have either $B \in C$ or $\neg B \in C$. If $\neg B \in C$, then we would have $M, w \models \neg B$, i.e. $M, w \forall \forall B$, which contradicts the fact that $M, w \models B$. Thus, we conclude $B \in C$.

The next lemma is the key for proving decidability of PPJ $\mathrm{JS}_{\mathrm{CS}}$. It completes the algorithm that we described from the beginning of the section by formally explaining how "justification and classical constraints" and "probabilistic constraints" can be satisfied in the same model. As it will be clear from the proof the formulas of the set $Y$ should all hold in worlds of some model. Hence, since the set $Y$ is finite Lemma 28 expresses a small model property. The $x_{i j}^{\prime} s$ in the Lemma should be understood as probabilities that are assigned to each of the worlds that satisfy a member of $Y$.

Lemma 28. Let CS be a constant specification and let $A$ be an $\mathcal{L}$-formula. $A$ is $\mathrm{PPJ} \mathrm{CS}_{\mathrm{C}, \text { Meas }}$-satisfiable if and only if there exists a non-empty set

$$
Y=\left\{B_{1}, \ldots, B_{n}\right\} \subseteq \operatorname{atoms}(A)
$$

such that all of the following conditions hold: 
1. for some $i \in\{1, \ldots, n\}, A \in B_{i}$.

2. for every $1 \leq i \leq n$, there exists an extended basic CS-evaluation that satisfies $B_{i}$.

3. for every $1 \leq i \leq n$, there are some $x_{i j}$ with $1 \leq j \leq n$, that satisfy the following linear equalities and inequalities:

$$
\begin{gathered}
\sum_{j=1}^{n} x_{i j}=1 \\
(\forall 1 \leq j \leq n)\left[x_{i j} \geq 0\right] \\
\text { for every } P_{\geq s} C \in B_{i}, \sum_{\left\{j \mid C \in B_{j}\right\}} x_{i j} \geq s \\
\text { for every } \neg P_{\geq s} C \in B_{i}, \sum_{\left\{j \mid C \in B_{j}\right\}} x_{i j}<s .
\end{gathered}
$$

Proof. Let CS be a constant specification and let $A \in \mathcal{L}$. We prove the two directions of the lemma separately:

$\Longrightarrow$ : Let $M=\langle U, W, H, \mu, *\rangle \in \mathrm{PPJ}_{\mathrm{CS}, \text { Meas }}$. Assume that $A$ is satisfiable in some world of $M$.

Let $\approx$ denote a binary relation over $U$ such that for all $w, x \in U$ we have:

$$
w \approx x \quad \text { if and only if } \quad(\forall B \in \operatorname{subf}(A))[M, w \models B \Leftrightarrow M, x \models B] .
$$

It is easy to see that $\approx$ is an equivalence relation. Let $K_{1}, \ldots, K_{n}$ be the equivalence classes of $\approx$ over $U$. For every $i \in\{1, \ldots, n\}$ we choose some $w_{i} \in K_{i}$. For every $i \in\{1, \ldots, n\}$ some subformulas of $A$ hold in the world $w_{i}$ and some do not. So, without loss of generality, we assume that for every $i \in\{1, \ldots, n\}$ there exists a $B_{i} \in$ atoms $(A)$ such that $M, w_{i} \models B_{i}$. For $i \neq j$ we have $B_{i} \neq B_{j}$ since $w_{i}$ and $w_{j}$ belong to different equivalence classes. Let $Y=\left\{B_{1}, \ldots, B_{n}\right\}$. Since $A$ holds in some $w_{i}, Y$ is non-empty. It remains to show that the conditions in the statement of the lemma hold:

1. Let $w \in U$ be such that $M, w \models A$. The world $w$ belongs to some equivalence class of $\approx$ that is represented by $w_{i}$. Thus $M, w_{i} \models A$. By Lemma 27 we find $A \in B_{i}$, i.e. condition 1 holds.

2. Let $i \in\{1, \ldots, n\}$. It holds that $M, w_{i} \models B_{i}$. We define the extended basic CS-evaluation $*_{i}$ as follows (the fact that $*_{i}$ is an extended basic CS-evaluation immediately follows from the fact that $*_{w_{i}}$ is a basic CSevaluation):

- for every $p \in$ Prop:

$$
p^{*_{i}}=p_{w_{i}}^{*}
$$


- for every $P_{\geq s} B \in \mathcal{L}$ :

$$
\left(P_{\geq s} B\right)^{*_{i}}= \begin{cases}\mathrm{T} & , \text { if } M, w_{i} \models P_{\geq s} B \\ \mathrm{~F} & , \text { if } M, w_{i} \not \models P_{\geq s} B ;\end{cases}
$$

- for every $t \in \mathrm{Tm}$ :

$$
t^{*_{i}}=t_{w_{i}}^{*} .
$$

The following statement can be shown by straightforward induction on the complexity of the formula.

$$
\left(\forall B \in \operatorname{subf}\left(B_{i}\right)\right)\left[M, w_{i} \models B \Longleftrightarrow *_{i} \models B\right]
$$

Since, $B_{i} \in \operatorname{subf}\left(B_{i}\right)$ and $M, w_{i} \models B_{i}$, by statement 5 we get $*_{i} \models B_{i}$. And of course this holds for every $1 \leq i \leq n$. Therefore, condition 2 holds.

3. Let $i \in\{1, \ldots, n\}$. We set

$$
y_{i j}=\mu\left(w_{i}\right)\left(K_{j} \cap W\left(w_{i}\right)\right), \text { for every } 1 \leq j \leq n .
$$

We are going to do some calculations to show that these values $y_{i j}$ satisfy the linear system in condition 3 .

First of all we have

$$
\begin{array}{lc}
\sum_{1 \leq j \leq n} y_{i j} & = \\
\sum_{1 \leq j \leq n} \mu\left(w_{i}\right)\left(K_{j} \cap W\left(w_{i}\right)\right) & \text { the } K_{j} \text { 's are mutually disjoint } \\
\mu\left(w_{i}\right)\left(\bigcup_{1 \leq j \leq n}\left(K_{j} \cap W\left(w_{i}\right)\right)\right) & \bigcup_{j=1}^{n} K_{j}=U \\
\mu\left(w_{i}\right)\left(W\left(w_{i}\right)\right) . &
\end{array}
$$

And since $\mu\left(w_{i}\right)$ is a finitely additive measure over $W\left(w_{i}\right)$ we get:

$$
\sum_{1 \leq j \leq n} y_{i j}=1
$$

By Eq. (6) we also have:

$$
(\forall 1 \leq j \leq n)\left[y_{i j} \geq 0\right] .
$$

Let $P_{\geq s} C \in B_{i}$. Since $M, w_{i} \models B_{i}$ it also holds that $M, w_{i} \models P_{\geq s} C$, i.e.

$$
\mu\left(w_{i}\right)\left([C]_{M, w_{i}}\right) \geq s .
$$


We will prove that:

$$
\bigcup_{\left\{j \mid C \in B_{j}\right\}}\left(K_{j} \cap W\left(w_{i}\right)\right)=[C]_{M, w_{i}} .
$$

Let $w \in[C]_{M, w_{i}}$. We have $w \in W\left(w_{i}\right)$ and $M, w \models C$. $w$ must belong to some $K_{j}$. We also have that $M, w_{j} \models C$ and $M, w_{j} \models B_{j}$, which by Lemma 27 implies $C \in B_{j}$. Thus, we proved that there exists some $j$ such that $C \in B_{j}$ and $w \in K_{j} \cap W\left(w_{i}\right)$. Thus

$$
w \in \bigcup_{\left\{j \mid C \in B_{j}\right\}}\left(K_{j} \cap W\left(w_{i}\right)\right) .
$$

On the other hand let $w \in \bigcup_{\left\{j \mid C \in B_{j}\right\}}\left(K_{j} \cap W\left(w_{i}\right)\right)$. So, there exists some $j$, such that $C \in B_{j}$ and $w \in K_{j} \cap W\left(w_{i}\right)$. It holds that $M, w_{j} \models B_{j}$ and since $w \in K_{j}$ we have that $M, w \models B_{j}$ which implies that $M, w \models C$. So, since $w \in W\left(w_{i}\right)$, we have that $w \in[C]_{M, w_{i}}$.

Therefore Eq. (10) holds.

By Eq. (9) and Eq. (10) we get:

$$
\mu\left(w_{i}\right)\left(\bigcup_{\left\{j \mid C \in B_{j}\right\}}\left(K_{j} \cap W\left(w_{i}\right)\right)\right) \geq s .
$$

Since the $K_{j}$ 's are mutually disjoint and $\mu\left(w_{i}\right)$ is a finitely additive measure we have:

$$
\sum_{\left\{j \mid C \in B_{j}\right\}} \mu\left(w_{i}\right)\left(K_{j} \cap W\left(w_{i}\right)\right) \geq s
$$

and by Eq. (6):

$$
\sum_{\left\{j \mid C \in B_{j}\right\}} y_{i j} \geq s
$$

So we proved that

$$
\text { for every } P_{\geq s} C \in B_{i}, \sum_{\left\{j \mid C \in B_{j}\right\}} y_{i j} \geq s .
$$

By a similar reasoning we can prove that

$$
\text { for every } \neg P_{\geq s} C \in B_{i}, \sum_{\left\{j \mid C \in B_{j}\right\}} y_{i j}<s .
$$

By Eqs. (7), (8), (11) and (12) we have that the $y_{i j}$ 's satisfy the linear system in condition 3. 
$\Longleftarrow$ : Assume that there exists some $Y=\left\{B_{1}, \ldots, B_{n}\right\} \subseteq$ atoms $(A)$ such that conditions $1-3$ in the lemma's statement hold. For every $1 \leq i \leq n$, let $*_{i}$ be an extended basic CS-evaluation such that $*_{i} \models B_{i}$ (by condition 2 we know that such an extended basic CS-evaluation exists). Let $x_{i j}$, for $i, j \in\{1, \ldots, n\}$, be numbers that satisfy the linear system in condition 3 .

We define the quintuple $M=\langle U, W, H, \mu, *\rangle$ as follows:

- $U=\left\{w_{1}, \ldots, w_{n}\right\}$ for some $w_{1}, \ldots, w_{n}$.

- For all $1 \leq i \leq n$ we set:

1. $W\left(w_{i}\right)=U$;

2. $H\left(w_{i}\right)=\mathcal{P}\left(W\left(w_{i}\right)\right)$;

3. for every $V \in H\left(w_{i}\right)$ :

$$
\mu\left(w_{i}\right)(V)=\sum_{\left\{j \mid w_{j} \in V\right\}} x_{i j}
$$

4. for every $p \in$ Prop:

$$
p_{w_{i}}^{*}=p_{i}^{*}
$$

and for every $t \in \mathrm{Tm}$ :

$$
t_{w_{i}}^{*}=t_{i}^{*} .
$$

First we show that $M \in \mathrm{PPJ}_{\mathrm{CS}, \text { Meas }}$. Since $Y$ is non-empty, $n$ is positive thus $U$ is non-empty too. Let $1 \leq i \leq n$. It holds that:

(i) $H\left(w_{i}\right)$ is an algebra over $W\left(w_{i}\right)$, since $H\left(w_{i}\right)$ is the powerset of $W\left(w_{i}\right)$.

(ii) For every $A \in \mathcal{L}$ we have that $[A]_{M, w_{i}} \in \mathcal{P}\left(W\left(w_{i}\right)\right)$, i.e. $[A]_{M, w_{i}} \in H\left(w_{i}\right)$.

(iii) $\mu\left(w_{i}\right)$ is defined for all $V \in H\left(w_{i}\right)$ and by the first two lines of the linear system in condition 3 it holds that the codomain of $\mu\left(w_{i}\right)$ is $[0,1]$.

We also have that:

$$
\mu\left(w_{i}\right)\left(W\left(w_{i}\right)\right)=\mu\left(w_{i}\right)(U)=\sum_{\left\{j \mid w_{j} \in U\right\}} x_{i j}=\sum_{1 \leq j \leq n} x_{i j}=1 .
$$

Let $U, V \in H\left(w_{i}\right)$ such that $U \cap V=\emptyset$. It holds

$$
\begin{aligned}
\mu\left(w_{i}\right)(U \cup V) & =\sum_{\left\{j \mid w_{j} \in U \cup V\right\}} x_{i j} \\
& =\sum_{\left\{j \mid w_{j} \in U\right\}} x_{i j}+\sum_{\left\{j \mid w_{j} \in V\right\}} x_{i j} \\
& =\mu\left(w_{i}\right)(U)+\mu\left(w_{i}\right)(V) .
\end{aligned}
$$

Thus, $\mu\left(w_{i}\right)$ is a finitely additive measure over $H\left(w_{i}\right)$. 
(iv) The fact that $*_{i}$ is an extended basic CS-evaluation immediately implies that $*_{w_{i}}$ is a basic CS-evaluation.

From (i) - (iv) we conclude that $M \in \mathrm{PPJ}_{\mathrm{CS} \text {, Meas }}$. It remains to show $M, w_{i} \models A$ for some $i$.

First we have to show the following statement:

$$
(\forall D \in \operatorname{subf}(A))(\forall 1 \leq i \leq n)\left[D \in B_{i} \Longleftrightarrow M, w_{i} \models D\right] .
$$

Let $D \in B_{i}$. We will prove statement (13) by induction on the structure of $D$. Let $1 \leq i \leq n$. We distinguish the following cases:

$D \equiv p \in$ Prop: It holds:

$\begin{array}{rlrl}D & \in B_{i} & & \Longleftrightarrow \\ p & \in B_{i} & & \Longleftrightarrow \\ *_{i} & =p & & \Longleftrightarrow \\ p^{*_{i}}=\mathrm{T} & & \Longleftrightarrow \\ p_{w_{i}}^{*}=\mathrm{T} & & \Longleftrightarrow \\ M, w_{i} & =p & & \Longleftrightarrow \\ M, w_{i} & =D & & \end{array}$

$D \equiv t: C:$ We have:

$\begin{aligned} D \in B_{i} & & \Longleftrightarrow \\ t: C \in B_{i} & & \Longleftrightarrow \\ *_{i} \models t: C & & \Longleftrightarrow \\ C \in t^{*_{i}} & & \Longleftrightarrow \\ C \in t_{w_{i}}^{*} & & \Longleftrightarrow \\ M, w_{i} \models t: C & & \\ M, w_{i} \models D & & \end{aligned}$

$D \equiv P_{\geq s} C$. We prove the two directions of the claim separately.

$\Longrightarrow$ : Assume that $D \in B_{i}$, i.e. $P_{\geq_{s}} C \in B_{i}$. By the third line of the linear system in condition 3 we have:

$$
\sum_{\left\{j \mid C \in B_{j}\right\}} x_{i j} \geq s .
$$

By the inductive hypothesis we have:

$$
\sum_{\left\{j \mid M, w_{j} \models C\right\}} x_{i j} \geq s
$$


It holds that

$$
[C]_{M, w_{i}}=\left\{w_{j} \in W\left(w_{i}\right) \mid M, w_{j} \models C\right\}=\left\{w_{j} \mid M, w_{j} \models C\right\} .
$$

By the definition of $M$ we have:

$$
\mu\left(w_{i}\right)\left([C]_{M, w_{i}}\right)=\sum_{\left\{j \mid w_{j} \in[C]_{M, w_{i}}\right\}} x_{i j} \stackrel{(15)}{=} \sum_{\left\{j \mid M, w_{j} \models C\right\}} x_{i j} .
$$

And by (14) we have that

$$
\mu\left(w_{i}\right)\left([C]_{M, w_{i}}\right) \geq s
$$

i.e.

$$
M, w_{i} \models P_{\geq s} C
$$

i.e.

$$
M, w_{i} \models D .
$$

$\Longleftarrow$ : Let $M, w_{i} \models D$. Assume that $D \notin B_{i}$, i.e. $\neg D \in B_{i}$, that is:

$$
\neg P_{\geq s} C \in B_{i} .
$$

By the last line of the linear system in condition 3 we have that

$$
\sum_{j: C \in B_{j}} x_{i j}<s .
$$

By using a similar argument as before we can prove that

$$
\mu\left(w_{i}\right)\left([C]_{M, w_{i}}\right)<s
$$

i.e.

$$
M, w_{i} \not \models D
$$

which is absurd. Therefore $D \in B_{i}$.

$D \equiv D_{1} \wedge D_{2}$ : Here $D \in B_{i}$ means

$$
D_{1} \wedge D_{2} \in B_{i} .
$$

We know that $*_{i} \models B_{i}$. Assume that $D_{1} \notin B_{i}$ or $D_{2} \notin B_{i}$. Then it should be $\neg D_{1} \in B_{i}$ or $\neg D_{2} \in B_{i}$, i.e. $*_{i} \nVdash D_{1}$ or $*_{i} \nVdash D_{2}$. But this is absurd since we have that $*_{i} \models D_{1} \wedge D_{2}$. So, both $D_{1}$ and $D_{2}$ belong to $B_{i}$. Hence (16) is equivalent to the following statements.

$$
\begin{array}{rlrl}
D_{1} \in B_{i} \text { and } D_{2} \in B_{i} & \stackrel{\text { i.h. }}{\Longleftrightarrow} \\
M, w_{i} \models D_{1} \text { and } M, w_{i} \models D_{2} & & \Longleftrightarrow \\
M, w_{i} \models D_{1} \wedge D_{2} & & \Longleftrightarrow \\
M, w_{i} \models D . & &
\end{array}
$$


$D \equiv \neg D^{\prime}:$ We have:

$\begin{array}{rlrl}D \in B_{i} & & \Longleftrightarrow \\ \neg D^{\prime} \in B_{i} & & \Longleftrightarrow \\ D^{\prime} \notin B_{i} & & \Longleftrightarrow \\ M, w_{i} \not \models D^{\prime} & & \Longleftrightarrow \\ M, w_{i} \models \neg D^{\prime} & & \Longleftrightarrow \\ M, w_{i} \models D . & \end{array}$

We conclude that statement (13) holds.

We have $A \in \operatorname{subf}(A)$. Thus, by statement (13) we find:

$$
(\forall 1 \leq i \leq n)\left[A \in B_{i} \Longleftrightarrow M, w_{i} \models A\right] .
$$

By condition 1 , there exists an $i$ such that $A \in B_{i}$. Thus, there exists an $i$ such that $M, w_{i} \models A$. Hence, $A$ is $\mathrm{PPJ}_{\mathrm{CS}, \text { Meas }^{-} \text {satisfiable. }}$

In the proof of Lemma 28 we construct a model with at most $2^{|\operatorname{subf}(A)|}$ worlds that satisfies $A$. Hence a corollary of Lemma 28 is that any $A \in \mathcal{L}$ is $\mathrm{PPJ}_{\mathrm{CS}, \text { Meas }^{-}}$ satisfiable if and only if it is satisfiable in a PPJ $\mathrm{CS}_{\text {,Meas }}$-model with at most $2^{\mid \text {subf }(A) \mid}$ worlds. In other words, as we already mentioned, Lemma 28 implies a

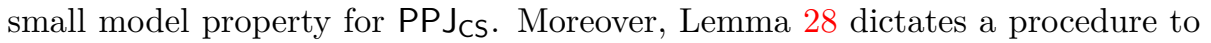
decide the satisfiability problem for $\mathrm{PPJ}_{\mathrm{CS}}$.

Theorem 29. Let CS be a decidable and schematic constant specification. The

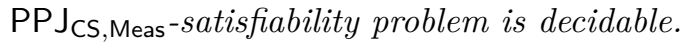

Proof. Let CS be a decidable almost schematic constant specification and let $A \in \mathcal{L}$. The formula $A$ is satisfiable if and only if for some $Y \subseteq$ atoms $(A)$ all conditions in the statement of Lemma 28 hold. Since atoms $(A)$ is finite, it suffices to show that for every $Y \subseteq$ atoms $(A)$ the conditions $1-3$ in the statement of Lemma 28 can be effectively checked:

- Decidability of condition 1 is trivial.

- Decidability of condition 2 follows from Lemma 25.

- In condition 3 we have to check for the satisfiability of a set of linear inequalities. There are several decision procedures available for this problem (see, for example, [24]).

We conclude that the PPJ $\mathrm{CS}_{\mathrm{C} \text { Meas-satisfiability problem is decidable. }}$ 


\section{$5 \quad$ Application to the Lottery Paradox}

Kyburg's famous lottery paradox [22] goes as follows. Consider a fair lottery with 1000 tickets that has exactly one winning ticket. Now assume a proposition is believed if and only if its degree of belief is greater than 0.99. In this setting it is rational to believe that ticket 1 does not win, it is rational to believe that ticket 2 does not win, and so on. However, this entails that it is rational to believe that no ticket wins because rational belief is closed under conjunction. Hence it is rational to believe that no ticket wins and that one ticket wins.

PPJ $J_{C S}$ makes the following analysis of the lottery paradox possible. First we need a principle to move from degrees of belief to rational belief (this formalizes what Foley [10] calls the Lockean thesis): we suppose that for each term $t$, there exists a term $\mathrm{pb}(t)$ such that

$$
t:\left(P_{>0.99} A\right) \rightarrow \mathrm{pb}(t): A
$$

(where pb stands for probabilistic belief). Let $w_{i}$ be the proposition ticket $i$ wins. For each $1 \leq i \leq 1000$, there is a term $t_{i}$ such that $t_{i}:\left(P_{=\frac{999}{1000}} \neg w_{i}\right)$ holds. Hence by (17) we get

$$
\operatorname{pb}\left(t_{i}\right): \neg w_{i} \quad \text { for each } 1 \leq i \leq 1000 .
$$

Now if CS is axiomatically appropriate, then

$$
s_{1}: A \wedge s_{2}: B \rightarrow \operatorname{con}\left(s_{1}, s_{2}\right):(A \wedge B)
$$

is a valid principle (for a suitable term $\operatorname{con}\left(s_{1}, s_{2}\right)$ ). Hence by (18) we conclude that

$$
\text { there exists a term } t \text { with } t:\left(\neg w_{1} \wedge \cdots \wedge \neg w_{1000}\right),
$$

which leads to a paradoxical situation since it is also believed that one of the tickets wins.

In PPJ $J_{C S}$ we can resolve this problem by restricting the constant specification such that (19) is valid only if con $\left(s_{1}, s_{2}\right)$ does not contain two different subterms of the form $\mathrm{pb}(t)$. Then the step from (18) to (20) is no longer possible and we can avoid the paradoxical belief.

This analysis is inspired by Leitgeb's [23] solution to the lottery paradox and his Stability Theory of Belief according to which it is not permissible to apply the conjunction rule for beliefs across different contexts. Our proposed restriction of (19) is one way to achieve this in a formal system. A related and very interesting question is whether one can interpret the above justifications $t_{i}$ as stable sets in Leitgeb's sense. Of course, our discussion of the lottery paradox is very sketchy but we think that probabilistic justification logic provides a promising approach to it that is worth further investigations. 


\section{Conclusion}

Extending the work of [15] we defined a probabilistic justification logic, PPJ, to study rational belief, degrees of belief and justifications. The logic PPJ is an extension of the probabilistic justification logic from [15] in the sense that PPJ allows iterations of the probability operators and justification operators over probability operators. In the framework of PPJ it is also possible to analyze a famous paradox from epistemology, i.e. the lottery paradox [22].

Furthermore, it is worth mentioning that heuristics have been applied for attacking the satisfiability problem for probabilistic logics with classical base [28]. It could be of practical interest to apply these heuristics to the satisfiability problem of probabilistic justification logic, too.

\section{References}

[1] Artemov, S.N.: Operational modal logic. Tech. Rep. MSI 95-29, Cornell University (Dec 1995)

[2] Artemov, S.N.: Explicit provability and constructive semantics. Bulletin of Symbolic Logic 7(1), 1-36 (Mar 2001)

[3] Artemov, S.N.: The logic of justification. The Review of Symbolic Logic 1(4), 477-513 (Dec 2008)

[4] Artemov, S.N.: The ontology of justifications in the logical setting. Studia Logica 100(1-2), 17-30 (Apr 2012), published online February 2012

[5] Artemov, S.N.: On aggregating probabilistic evidence. In: Artëmov, S.N., Nerode, A. (eds.) Logical Foundations of Computer Science - International Symposium, LFCS 2016, Deerfield Beach, FL, USA, January 4-7, 2016. Proceedings. Lecture Notes in Computer Science, vol. 9537, pp. 27-42. Springer (2016)

[6] Artemov, S.N., Fitting, M.: Justification Logic: Reasoning with Reasons. Cambridge University Press (2019)

[7] Bucheli, S., Kuznets, R., Studer, T.: Partial realization in dynamic justification logic. In: Beklemishev, L.D., de Queiroz, R. (eds.) Proc. WoLLIC 11, LNAI, vol. 6642, pp. 35-51. Springer (2011)

[8] Fan, T., Liau, C.: A logic for reasoning about justified uncertain beliefs. In: Yang, Q., Wooldridge, M. (eds.) Proc. IJCAI 2015. pp. 2948-2954. AAAI Press (2015)

[9] Fitting, M.: The logic of proofs, semantically. Annals of Pure and Applied Logic 132(1), 1-25 (Feb 2005) 
[10] Foley, R.: Beliefs, Degrees of Belief, and the Lockean Thesis. In: Huber, F., Schmidt-Petri, C. (eds.) Degrees of Belief, pp. 37-47. Springer (2009)

[11] Ghari, M.: Justification logics in a fuzzy setting. ArXiv e-prints (Jul 2014)

[12] Ghari, M.: Pavelka-style fuzzy justification logics. Logic Journal of the IGPL 24(5), 743-773 (2016), https://doi.org/10.1093/jigpal/jzw019

[13] Kokkinis, I.: The complexity of non-iterated probabilistic justification logic. In: Gyssens, M., Simari, G.R. (eds.) Foundations of Information and Knowledge Systems - 9th International Symposium, FoIKS 2016, Linz, Austria, March 7-11, 2016. Proceedings. Lecture Notes in Computer Science, vol. 9616, pp. 292-310. Springer (2016)

[14] Kokkinis, I.: The complexity of satisfiability in non-iterated and iterated probabilistic logics. Annals of Mathematics and Artificial Intelligence 83(34), 351-382 (2018)

[15] Kokkinis, I., Maksimović, P., Ognjanović, Z., Studer, T.: First steps towards probabilistic justification logic. Logic Journal of IGPL 23(4), 662-687 (2015)

[16] Kuznets, R.: On the complexity of explicit modal logics. In: Clote, P.G., Schwichtenberg, H. (eds.) Proc. CSL 2000, LNCS, vol. 1862, pp. 371-383. Springer (2000)

[17] Kuznets, R.: Complexity Issues in Justification Logic. Ph.D. thesis, City University of New York (May 2008), http://gradworks.umi.com/33/10/ 3310747.html

[18] Kuznets, R., Studer, T.: Justifications, ontology, and conservativity. In: Bolander, T., Braüner, T., Ghilardi, S., Moss, L. (eds.) AiML 9, pp. 437458. College Publications (2012)

[19] Kuznets, R., Studer, T.: Update as evidence: Belief expansion. In: Artemov, S.N., Nerode, A. (eds.) Proc. LFCS 2013, LNCS, vol. 7734, pp. 266279. Springer (2013)

[20] Kuznets, R., Studer, T.: Weak arithmetical interpretations for the logic of proofs. Logic Journal of IGPL 24(3), 424-440 (2016)

[21] Kuznets, R., Studer, T.: Logics of Proofs and Justifications. College Publications (2019)

[22] Kyburg, H.E.J.: Probability and the Logic of Rational Belief. Wesleyan University Press (1961)

[23] Leitgeb, H.: The stability theory of belief. Philosophical Review 123(2), $131-171(2014)$ 
[24] Luenberger, D.G., Ye, Y.: Linear and Nonlinear Programming, International Series in Operations Research and Management Science, vol. 116. Springer (2008)

[25] Milnikel, R.S.: The logic of uncertain justifications. Annals of Pure and Applied Logic 165(1), 305-315 (2014)

[26] Mkrtychev, A.: Models for the logic of proofs. In: Adian, S., Nerode, A. (eds.) Proc. LFCS 1997, LNCS, vol. 1234, pp. 266-275. Springer (1997)

[27] Ognjanović, Z., Rašković, M.: Some first order probability logics. Theoretical Computer Science 247, 191-212 (2000)

[28] Ognjanović, Z., Rašković, M., Marković, Z.: Probability logics. Zbornik radova, subseries "Logic in Computer Science" 12(20), 35-111 (2009)

[29] Rašković, M., Ognjanović, Z.: A first order probability logic, $L P_{Q}$. Puplications de L'Institut Mathèmatique 65(79), 1-7 (1999)

[30] Stepić, A.I., Ognjanović, Z.: Complex valued probability logics. Publications de l'Institut Mathématique (N.S.) 95(109), 73-86 (2014)

[31] Studer, T.: Decidability for some justification logics with negative introspection. Journal of Symbolic Logic 78(2), 388-402 (Jun 2013) 